\title{
Teaching optics in electrical engineering curriculums
}

\author{
Alexander Poularikas, Samuel Seely
}

Alexander D. Poularikas, Samuel Seely, "Teaching optics in electrical engineering curriculums," Proc. SPIE 1603, Education in Optics, (1 March 1992); doi: $10.1117 / 12.57852$ 
Teaching optics in electrical engineering curriculums

by

\author{
Alexander D Poularikas and Samuel Seely \\ University of Alabama in Huntsville \\ Electrical and Computer Engineering Department \\ Huntsville, Alabama 35899
}

\begin{abstract}
A program is proposed that alleviates to some extent the educational problems created by the introduction of the many new technical areas into electrical engineering. It presumes the time-tested philosophy of circular learning, namely, successive review and extension of knowledge and understanding with successive courses. We propose a one year program that provides a broad overview of the foundations to all subsequent courses; thus included within this program are the first essentials of a wide variety of later studies. In addition, it is proposed to divide the science of electrical engineering into broad fundamental areas which will be made up of the appropriate parts that are taught today separately. Included, as a fundamental concept in this scheme, is also proposed to embed in the already existing areas any new diverse topic such as optics, superconductivity, etc. In the present study we have used the optics to test our new philosophy on engineering education.
\end{abstract}

\title{
1. INTRODUCTION
}

A basic problem that has faced engineering educators during the last half of the 20th century is how to adapt their particular curriculums to the technical developments that have occurred during this ensuing period. How much of each major development and how to include the requisite studies has been a continuous challenge. This need has been accomplished in various ways since all curriculums are faced with constraints on the number of courses and the number of credit hours that are physically and educationally possible and available to a department within its normal college program.

It has been our experience and general observation that the usual approach is to rearrange course content, by changing course requirements, and by substituting a new sequence of courses, often at the expense of basic courses covering the principles on which general understanding depends. Often, these methods have caused communication gaps since they assume a level of student sophistication at a given time which may not have been attained in previous studies. All too frequently the student must tell the professor that the assumption that he had previously studied certain material basic to the new program is not valid. This lack of sequence might result from the lack of careful coordination among courses within a department. It might also result from the educational gaps that are inherent within departmental offerings.

We will, in what follows, limit our discussion to an approach taken by the authors in a plan for electrical engineering education. This plan provides a first step in a coordinated learning experience.

\section{THE CIRCULAR PLAN}

A listing of the areas of concern to electrical engineering education is as impressive as it is extensive. Yet we blithely proceed with the view that the student is expected, after his four year program, to have a working knowledge of such areas as: Networks, devices, system theory, computer circuits and engineering, discrete time systems and signals, electronics, field theory, communications, electromechanical transducers and energy conversion devices, control engineering, and perhaps even bioengineering, robots, and optical engineering. It is the purpose of this paper to suggest a plan that provides a base for accomplishing this educational objective. The problem is aggravated by the need to recognize at the outset that these studies must be consistent with other studies required of the student and with the limited time and experience of the student in his first year of college mathematics and physics. Obviously, however this educational problem is attacked, it must initially limit itself to fundamental ideas 
and principles and then to show how these are involved in the many area of electrical engineering. An essential need is also that of introducing the student to some of the varied and often specialized terminology that has developed within these separate areas. In essence, what is initially needed is displaying horizons rather than with the subtleties that will be included ultimately in the student's educational career.

Our proposed attack on this educational problem is patterned in part on the philosophy adopted by physics departments in their usual approach to the study of physics. This ia a circular approach, with a rather thin level of sophistication in the first or general introductory course or courses. A large number of books have been written for this general, first level, study. The level of this first study can vary over wide limits depending on the prerequisites imposed, usually that the student has completed a general physics program in high school. Following this first introductory level study, each sub-area can be given further treatment in one or more specialized courses. This requires a number of second level courses, but these can proceed with the knowledge that the student has already been introduced to the concepts of the field. Thus the total study by the student will consist of the introductory sequence followed by courses, the number and depth of these courses depending upon the area of special interests of the students. Clearly, the scope and level of understanding comes with successive expansion of studies in each of the sub-areas. If the mathematical demands are beyond the required formal studies in mathematics, these must be included as integral parts of the new study.

Our proposed educational plan for electrical engineering students begins at the second semester of the Freshman year, if the students background in mathematics extends well into ihe calculus. Otherwise, the plan would begin at the start of the Sophomore year. This plan is for a one-year sequence that provides a reasonably balanced presentation of the essential areas of electrical engineering. The level should be adequate for a reasonable understanding of each sub-area and the interrelationship among sub-areas with the fundamental aspects of basic principles being stressed and highlighted.

We wish to detail the broad outline for such an introductory sequence, as we have developed it.

\section{Introduction and Concepts of Electrical Engineering}

The study consists of a number of related segments, with later segments dealing specifically with areas of specialization. This introductory sequence is to be accompanied by laboratory work appropriate to the course content. An outline of the basic course content follows:

1. Signals, Models and Modeling. Discusses the general problem of describing in mathematical terms the models of the physical components and the important signal wave shapes that constitute the real world-mathematical world interface that engages the attention of the electrical engineer.

2. Writing Equations for Interconnected Systems. Establishes systematic methods for writing the equations that describe interconnected systems of physical elements. Essentially a study of the Kirchhoff laws for electrical circuits, with some attention to the corresponding D'Alembert principles for discussing mechanical systems.

3. System Response: Time Domain Considerations. The transient and steady state response of simple systems to a variety of input forcing functions. This involves the use of certain fundamental aspects of linear differential equations.

4. System Response to Sinusoidal Excitation Functions. Discusses the response of systems to sinusoidal time excitation functions. This is, therefore, the essentials of ac circuit analysis.

5. the Laplace Transform. Emphasizes the concept of mathematical transformations from the time domain to a complex frequency plane domain as a means for deducing the time domain solution of differential equations. 
6. Signal Flow Graphs, Block Diagrams and Transfer Functions. Presents the important ways of graphically portraying an interconnected system in order to highlight the signal path from input to the output of the system. It provides a convenient means for introducing the concepts of feedback.

7. Electronic Circuits. A study of basic principles applied to electronic devices and circuits. Device modeling is emphasized. Attention is directed to bipolar junction transistors in both large signal and incremental mode operation. Field effect transistors of various types are studied, with considerations of the behavior of different classes and types of FET's. Circuit integration is discussed.

8. Measurements and Instruments. A review of the fundamentals of electrical measurements with emphasis on basic equipment in general use. Includes a discussion of estimates of accuracy of measurements and the theory of errors.

9. Digital Systems. An introduction to digital devices and systems as they relate to digital circuits, the digital computer, and the microprocessor.

10. Discrete Time Signals and Systems. Discrete time considerations in modeling and analysis. The mathematical formulation leads naturally into numerical methods of analysis. It also permits the introduction of Z-transform methods to solve the difference equations that describe such systems. The concepts of digital filtering are introduced in this study.

11. Waves and Radiation. The basics of wave phenomena in space with applications in diverse fields, including radiation, transmission, diffraction and modern optics and electrooptics.

12. Communication Systems. The basic concepts of communications under continuous, sampled and digital modulation and demodulation methods.

13. Electromechanics. A review of the important aspects of electricity and magnetism, followed by a discussion of energy converters, incremental transducers, dc and ac rotating machinery. The operating and starting characteristics of the more common rotating machines are discussed.

14. Automatic Control. Expands certain prior studies to a consideration of feedback principles with applications to feedback control systems. Includes a discussion of the methods of compensation in system design to meet prescribed specifications.

After the student has been introduced to the fundamentals of electrical engineering he/she will be ready to embark on studies in more depth in the different areas of their discipline. The fundamental philosophy of this proposal is that it will serve as the platform for extended and new educational demands.

The scope and content of the proposed circular study based plan is very broad. However, it is very important to realize that we are discussing only the initial stage of a larger study, that is, this is an introductory survey and should be viewed as a first effort to introduce broad concepts, and is not an effort to provide a complete electrical engineering education. As developed, the content can actually fulfill a variety of educational needs. The first six sections can be completed in a one-semester course, and can, in fact, serve all engineering students, as an introduction to electrical engineering; it is not directed exclusively to electrical engineering students. The remaining 8 sections can provide the background for a variety of follow-up programs. For example: Secs. 7, 8, 9, 10 can be considered as a basic to the study of digital systems; Secs. 7, 8, 10,12, 13 would provide the necessary background for further studies in control systems; Secs. 7, 8, 10,11, 12 would provide the desired background for additional studies in communication systems. Other groupings are possible as background studies for any reasonable subsequent course or program objectives within an electrical engineering curriculum. 


\section{THE PROPOSED EE CURRICULUM}

We propose that the total field of Electrical Engineering be separated into four broad areas. Within each area the appropriate learning material will be included. It is also proposed that a unified approach in the development of material be implemented. By unified approach we mean that cross references by examples and problems of those disciplines belonging to the broad area are freely used. In other words, we visualize a continuum rather than a subject by subject modules separation as is done today. In addition, every effort should be made in the development of the material so that whenever appropriate, some examples from areas outside the Electrical Engineering discipline are incorporated. This pedagogical tool provides the student with confidence that the fundamentals he is learning in his field can also be applied to solve problems in other fields.

As time progresses and new subdisciplines appear they can be easily incorporated in these broad areas by adding new material or new problems or new examples or all of the above. This type of implementation we have called the embedded method and it is elaborated in the next section. The proposed areas of Electrical Engineering curriculum is shown in Fig. 1. In addition to those subdisciplines shown in Fig. 1, the student should acquire ability to work with at least two computer languages, such as Fortran, C, or Pascal. The student must also develop a mathematical background that includes the following subjects: Differential Equations, Complex Varibles, Linear Algebra, Orthogonal Functions and Transforms, Random Processes.

\section{THE EMBEDDED PLAN}

To investigate the effectiveness of our proposed curriculum changes, we consider the area of optics as the test case for the embedded method. With Secs. 11, 12, 14 as background we can proceed towards this goal using two books we have already written and third is in planning stages.

We identify three basic areas of optics and the corresponding emphasis areas that are now taught widely in the EE curriculums and which constitute the basic knowledge required by the student to learn about optics. These are (see also Fig. 2):

\section{OPTICS}

Geometric and

Physical Optics

Fiber Optics

Fourier Optics

Optical Signal Processing

Optical Computing

Optical Communications

\section{EE COURSES}

Electromagnetic Theory

(Electromagnetics: Classical and Modern

Theory and Applications. S. Seely and A.

D. Poularikas, Marcel Dekker, New York, N.Y. 1979)

Signals and Systems

(Signals and Systems. A.D. Poulrikas and S. Seely. 2nd Edition, PWS-KENT, Boston, MA., 1991)

Communications.

(Text in preparation)

In our Electromagnetic Theory book we have introduced the following topics from the field of optics: Crystal Optics; Geometrical Optics Approximation for EM Waves; Light Rays; Fermat's Principle; Imaging in Refracting Media; Aberration Coefficients; Propagation of Optical Beams; Fiber Optics; Rays Guided by Lenses; Image Formation in Gaussian Optics; Diffraction Theory; Faunhofer Region and Fourier Transform of Fields; Diffraction Holography; Gaussian Light Beam; Coherence of Fields; Fabry-Perot Resonator Optics; Optical Resonators; Stability Diagram of Optical Resonators. 
In the Signals and System book we have introduced the following additional topics from the field of optics: Fourier Transforms of Optical Systems; Modulation of Light; Optical System Functions; Spread Function; Optical Transfer Function; Frequency Response of Coherent System; Frequency Response of Incoherent System; Optical Filtering; Optical Correlation; Phase and Amplitude Filters; Vander Lugt Filter.

In the Communications book we plan to introduce the following additional topics from the field of Optics: Optical Fiber Measurements; Optical Sources; Optical Detectors; Optical Receiver Noise Considerations; Optical Transmitters; Optical Receivers; Optical Modulation Schemes; Optical Transducers.

\section{CONCLUSIONS}

The proposed circular plan provides an overview of most important areas of modern electrical engineering. It is only the first step in the learning experience, and presupposes further extension of the important details of any particular area of concern. It provides sufficient depth and understanding at the early level so that future in-depth studies will take a recognized and understandable place in the total learning experience of the student.

The need to separate the EE discipline into four major areas with the unified approach implemented is to recognize that technology progresses very fast and often the details of every new subject must wait to be learned after the bachelor's degree. Therefore, the student must be exposed to the fundamentals of each area in order to acquire the necessary tools to further his work in his later years. Furthermore, this approach eliminates the need for core courses.

The embedded approach will alleviate the problem of almost regularly having to introduce new courses into the EE curriculum and at the same time will satisfy a great pedagogical need, the need to show that learning the fundamentals is paramount in education and that you can use the information in many seemingly diverse fields.

The embedded method is: a) flexible, since you can add and or subtract material as new items become more important, b) Universal, since it can be applied to any discipline old or new, c) Highly pedagogical, since it brings in the fundamentals of any area of study, d) Interdisciplinary, since the material of one area appears in many areas of study.

The combination of the Circular, the Subdivision and Embedded plans can be considered as fundamentally powerful pedagogical vehicles to transmit not only the appropriate but also the most important and fundamental information. It must be well understood by the educators that in a 4-year program we can't teach details and must, therefore, concentrate in providing the most fundamental laws, ideas, approaches and methods. This is very important to young students who enter our schools today because at the same time they enter an era of life-long learning, and if the students do not receive a good foundation, their learning abilities at a later date will be severely hampered. They will lack the "Head-Start Program."

The feature of the proposed plan is that it allows a presentation of the basics of the entire electrical engineering field without gaps and without duplications that inevitably occur when traditional courses begin with certain assumptions of prior background knowledge and experience.

It has, therefore, been shown that we can successfully teach optics to electrical engineering students without introducing new courses or add additional credit hours. 


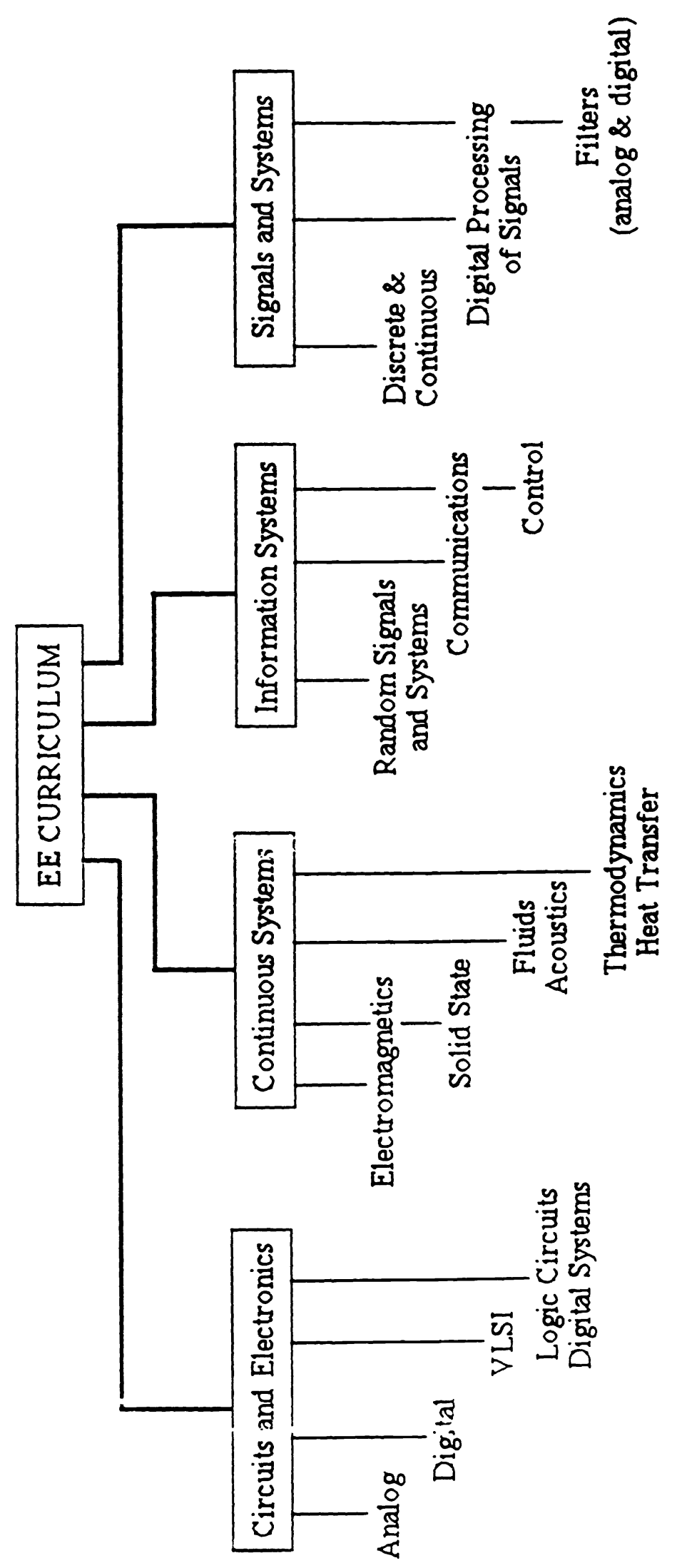

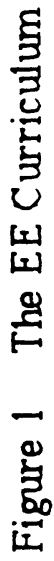




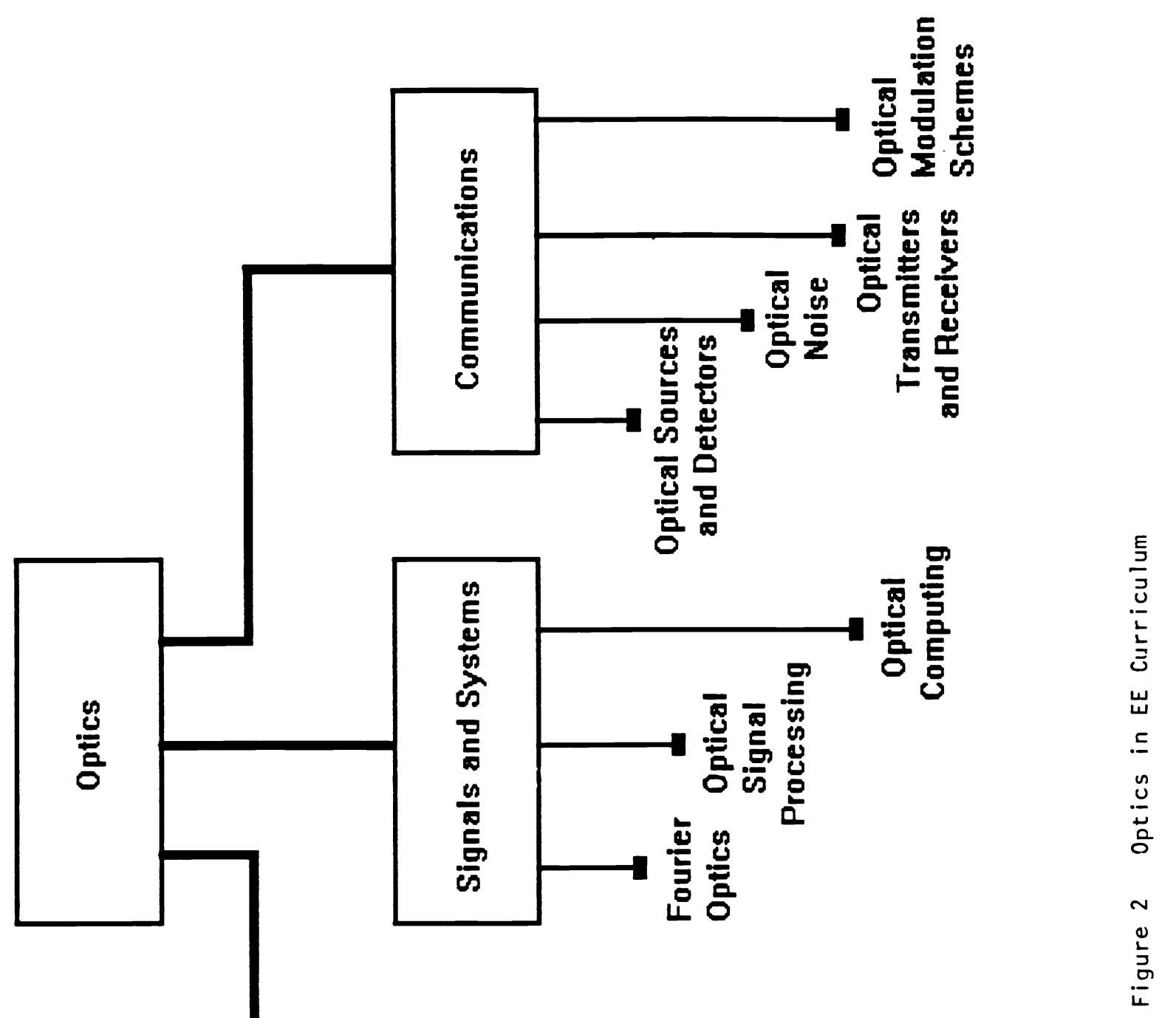

